\title{
Video Article \\ Isolation and Culture of Individual Myofibers and their Satellite Cells from Adult Skeletal Muscle
}

\author{
Alessandra Pasut ${ }^{1,2}$, Andrew E. Jones ${ }^{1,2}$, Michael A. Rudnicki ${ }^{1,2}$ \\ ${ }^{1}$ Sprott Center for Stem Cell Research, Ottawa Hospital Research Institute \\ ${ }^{2}$ Department of Cellular and Molecular Medicine, University of Ottawa \\ Correspondence to: Michael A. Rudnicki at mrudnicki@ohri.ca
}

URL: https://www.jove.com/video/50074

DOI: doi:10.3791/50074

Keywords: Stem Cell Biology, Issue 73, Cellular Biology, Molecular Biology, Medicine, Biomedical Engineering, Bioengineering, Physiology, Anatomy, Tissue Engineering, Stem Cells, Myoblasts, Skeletal, Satellite Cells, Skeletal Muscle, Muscular Dystrophy, Duchenne, Tissue Culture Techniques, Muscle regeneration, Pax7, isolation and culture of isolated myofibers, muscles, myofiber, immunostaining, cell culture, hindlimb, mouse, animal model

Date Published: $3 / 22 / 2013$

Citation: Pasut, A., Jones, A.E., Rudnicki, M.A. Isolation and Culture of Individual Myofibers and their Satellite Cells from Adult Skeletal Muscle. J. Vis. Exp. (73), e50074, doi:10.3791/50074 (2013).

\section{Abstract}

Muscle regeneration in the adult is performed by resident stem cells called satellite cells. Satellite cells are defined by their position between the basal lamina and the sarcolemma of each myofiber. Current knowledge of their behavior heavily relies on the use of the single myofiber isolation protocol. In 1985, Bischoff described a protocol to isolate single live fibers from the Flexor Digitorum Brevis (FDB) of adult rats with the goal to create an in vitro system in which the physical association between the myofiber and its stem cells is preserved ${ }^{1}$. In 1995 , Rosenblattmodified the Bischoff protocol such that myofibers are singly picked and handled separately after collagenase digestion instead of being isolated by gravity sedimentation ${ }^{2,3}$. The Rosenblatt or Bischoff protocol has since been adapted to different muscles, age or conditions ${ }^{3-6}$. The single myofiber isolation technique is an indispensable tool due its unique advantages. First, in the single myofiber protocol, satellite cells are maintained beneath the basal lamina. This is a unique feature of the protocol as other techniques such as Fluorescence Activated Cell Sorting require chemical and mechanical tissue dissociation ${ }^{7}$. Although the myofiber culture system cannot substitute for in vivo studies, it does offer an excellent platform to address relevant biological properties of muscle stem cells. Single myofibers can be cultured in standard plating conditions or in floating conditions. Satellite cells on floating myofibers are subjected to virtually no other influence than the myofiber environment. Substrate stiffness and coating have been shown to influence satellite cells' ability to regenerate muscles ${ }^{8,9}$ so being able to control each of these factors independently allows discrimination between niche-dependent and -independent responses. Different concentrations of serum have also been shown to have an effect on the transition from quiescence to activation. To preserve the quiescence state of its associated satellite cells, fibers should be kept in low serum medium ${ }^{1-3}$. This is particularly useful when studying genes involved in the quiescence state. In serum rich medium, satellite cells quickly activate, proliferate, migrate and differentiate, thus mimicking the in vivo regenerative process ${ }^{1-3}$. The system can be used to perform a variety of assays such as the testing of chemical inhibitors; ectopic expression of genes by virus delivery; oligonucleotide based gene knock-down or live imaging. This video article describes the protocol currently used in our laboratory to isolate single myofibers from the Extensor Digitorum Longus (EDL) muscle of adult mice (6-8 weeks old).

\section{Video Link}

The video component of this article can be found at https://www.jove.com/video/50074/

\section{Protocol}

All experiments were handled according to the University of Ottawa regulations for animal care and handling.

See Table 1 for an overview of the protocol.

\section{Before Starting the Isolation}

1. Prepare the following solutions:

$0.2 \%$ Collagenase type I in DMEM (Dulbecco's modified Eagle's medium; high glucose, L-glutamine with $110 \mathrm{mg} / \mathrm{ml} \mathrm{sodium} \mathrm{pyruvate).}$

For two EDL muscles prepare $2 \mathrm{ml}$ of $0.2 \%$ Collagenease in DMEM. Filter the solution through $0.22 \mu \mathrm{m}$ filter. 10 min before the isolation, prewarm at $37^{\circ} \mathrm{C}$ in a water bath. Additional aliquots of undiluted collagenase can be frozen at $-20^{\circ} \mathrm{C}$ for later use.

Note: use DMEM with sodium pyruvate throughout all of the procedure. Fibers do not survive in sodium pyruvate-free medium.

- Washing media (use to perform all the washes). Supplement DMEM with $1 \%$ Penicillin/Streptomycin. Filter through $0.22 \mu \mathrm{m}$ filter before use.

- Myofiber culture media. Supplement DMEM with 20\% FBS, 1\% Chicken Embryo extract and 1\% Penicillin/Streptomycin. Filter through $0.22 \mu \mathrm{m}$ filter before use. 
- Matrigel coated dishes. To culture fibers for long period of time, we recommend using Matrigel as coating substrate. To prepare Matrigel coated dishes, thaw an aliquot of Matrigel at $4{ }^{\circ} \mathrm{C}$ overnight. The day after, dilute Matrigel 1:10 in DMEM. Keep Matrigel at $4{ }^{\circ} \mathrm{C}$ at all times and avoid abrupt temperature changes as this will create microscopic crystals within the Matrigel solution resulting in uneven coating. Place dishes to be coated on ice. Coat dishes with just enough volume to cover the surface. Let it sit for $1 \mathrm{~min}$. Completely remove the leftover Matrigel. Let dishes dry at $37^{\circ} \mathrm{C}$ for at least $3 \mathrm{hr}$ prior to use or overnight.

Note: aliquots of diluted Matrigel can be re-used multiple times for coating purpose if stored at $4{ }^{\circ} \mathrm{C}$.

2. Lastly, be sure to clean the microscope station and dissecting tools with $70 \%$ ethanol.

3. For two EDL isolations (one mouse) prepare five plastic Petri dishes $(60 * 15 \mathrm{~mm})$ as follows:

- Four dishes for the isolation (1 for muscle dissociation, 3 for serial washes). All dishes must be coated with horse serum (HS) to prevent myofibers from attaching to plastic. To coat, pipette $3 \mathrm{ml}$ of horse serum into each dish, swirl to allow even coating, remove the horse serum and let the dish dry for at least $30 \mathrm{~min}$. Add $4 \mathrm{ml}$ of DMEM to each dish. Keep dishes at $37^{\circ} \mathrm{C}$ in a $5 \% \mathrm{CO}_{2}$ incubator prior to use.

Note: Horse serum can be re-used multiple times, if kept sterile. Alternatively, a solution of $10 \% \mathrm{HS}$ in DMEM can be used to coat dishes. Use HS coated dishes throughout the whole protocol and when culturing myofibers on floating conditions.

- One dish will be used to culture myofibers after the isolation. Alternative dish sizes or formats can be used for the final culture of myofibers depending on downstream applications. However, we do not suggest dish sizes larger than $60 * 15 \mathrm{~mm}$. Myofibers can be kept in suspension for no longer than $96 \mathrm{hr}$ before hyper contraction occurs. For longer culture times, we recommend using Matrigel coated dishes or plates.

4. For one fiber isolation (2 EDL), prepare two sterile Pasteur pipettes: one large bore pipette for muscle handling and one small bore pipette for myofiber manipulation. Use a diamond pen to cut each glass pipette to the desired length and heat polish to smooth pipette's edges. By using the flame, curve the tip of the small bore pipette. This will help handling single fibers. Flame to sterilize. Coat each pipette with HS before use.

\section{Muscle Dissection and Digestion}

1. For the purpose of these experiments, 8 week old SV129, Pax7 CreER ${ }^{\text {Cklr }}$;Rosa26TdTomato (Pax7Cre-TdTomato) and Myf5-Cre;Rosa26YFP were used.

2. Spray hind limbs with $70 \%$ ethanol. Pin the animal (face up) to a support board to have a better grasp of the hind limb during the procedure.

3. With the help of scissors, cut through the entire length of the limb and expose the underlying muscle. Remove the skin as well as any hair or fur (Figure 1A).

4. With a fine scissor, cut through the thin fascia without damaging the underlying muscles. Visually localize the EDL. The EDL is found in the anterior compartment of the hind limb just underneath the Tibialis Anterior (TA) muscle.

5. With the help of two forceps, expose the distal tendons.

6. Cut the distal tendons (of both the TA and EDL) with sharp Cohann-Vannas spring scissors.

7. With the help of forceps hold both TA and EDL muscles by their tendons and delicately pull the muscles up towards the proximal end. At this point you should be able to clearly see the EDL muscle just underneath the TA muscle. Now, separate the EDL from the TA muscle by pulling the two tendons in opposite directions (Figure 1B). Avoid stretching the EDL muscle while performing this operation as this will damage the myofibers.

8. Expose the EDL tendon. To better visualize the proximal tendon it may help at this point to remove the TA muscle. It may also help to cut off some the connective tissue around the knee (Figure 1C).

9. Cut the proximal tendon and gently remove the EDL (Figure 1D).

10. By holding the muscle through its tendons, transfer it to $2 \mathrm{ml}$ of previously prepared collagenase solution. Incubate at $37^{\circ} \mathrm{C}$ in a water bath. Note: for successful fiber isolation, it is important to isolate the EDL from tendon to tendon so that the myofiber integrity is maintained. Steps 2.3 to 2.9 can be performed under a dissecting microscope. Alternatively, the use of a magnifying glass may also help to have a better view of the muscles.

11. Repeat steps 2.3 to 2.9 to isolate the second EDL. Transfer the second EDL in the same tube. To avoid uneven digestion, isolation of the second EDL should be completed no more than 5 min after the first.

Note 1: incubation time may need to be adjusted depending on collagenase activity. Longer or shorter incubation might be required depending on the size, age and/or muscle condition (e.g. fibrotic muscles need longer digestion time).

Note 2: If examining satellite cells behavior under quiescence conditions, agitation during muscle digestion time may activate satellite cells ${ }^{10}$

12. During the digestion time, regularly check the muscle to avoid over-digestion. Stop the digestion when muscles start to loosen up and myofibers are visible. To stop digestion, carefully transfer both muscles to a prewarmed Petri dish with $4 \mathrm{ml}$ of DMEM (dissociation dish). Use the large bore size pipette to perform this operation.

Note: avoid muscle overdigestion as this inevitably results in the isolation of hyper contracted myofibers.

\section{Single Myofiber Dissociation and Culture}

1. To release myofibers, use the large bore glass pipette to flush the muscle with warm medium until fibers naturally start being released. Do not triturate the muscle as this will inevitably result in damaging fibers. Perform this and the following steps under a dissecting microscope.

2. Continue releasing myofibers until the desired number is reached. If the dish is required at room temperature for more than 10 min allow a 5 min (minimum) incubation at $37{ }^{\circ} \mathrm{C}, 5 \% \mathrm{CO}_{2}$ to re-equilibrate the medium.

Note: if medium reaches temperatures below physiological $\left(37^{\circ} \mathrm{C}\right)$ for an extended time myofibers will die.

3. Using the small size bore pipette, transfer live single myofibers to a new prewarmed dish (first of three consecutive washes). Handle each myofiber individually instead of transferring bulk of myofibers all at once. If necessary, incubate at $37{ }^{\circ} \mathrm{C}, 5 \% \mathrm{CO}_{2}$ for $10-15$ min to reequilibrate the medium.

4. Repeat step 3.3 for 2 more times or until all dead myofibers and debris are removed. We recommend at least three consecutive washes for proper clean-up. 
Note: dead myofibers will appear as short and hyper contracted under the microscope light.

5. Incubate single myofibers at $37^{\circ} \mathrm{C}, 5 \% \mathrm{CO}_{2}$ in the last wash dish (DMEM only) for at least one hr prior to switching to culture medium. This allows myofibers to adjust to the in vitro conditions in the absence of serum. We found that immediate culture of myofibers in serum rich medium increases fiber shrinking.

6. After one hr, transfer myofibers to a new prewarmed dish or to the appropriate culture format depending on the downstream application. Culture fibers in high serum medium to allow satellite cell activation. Alternatively, different concentrations of serum or chicken embryo extract can also be used. Change medium every other day.

\section{Downstream Applications}

1. Immunostaining. Live myofibers can be fixed and stained at any time point during the isolation. Immunofluorescence can be performed on both floating and substrate-attached myofibers. If staining floating myofibers, use a small bore glass pipette to transfer myofibers from one solution to another. Alternatively, it is possible to keep myofibers in the same well throughout all the procedure and add/remove solutions using a glass pipette. Avoid standard aspiration as this will result in the removal of the myofibers as well. Briefly, completely remove culture medium; fix myofibers in prewarmed 4\% paraformaldehyde (PFA) for 5 min. Extensively wash in PBS several times. Incubate myofibers in $1 \%$ glycine in PBS or other standard quenching solutions to minimize PFA background staining. If necessary, permeabilize myofibers with $0.1 \%$ Triton X-100 in PBS for 10 min followed by a 5 min wash in PBS. Incubate fibers in blocking solution (10\% horse serum, $0.1 \%$ Triton $\mathrm{X}-100,1 \% \mathrm{NaN}$ ) for $1 \mathrm{hr}$ at room temperature or preferably overnight at $4{ }^{\circ} \mathrm{C}$. Alternative blocking solutions may be used, depending on the antibody of interest. Wash once in PBS for $5 \mathrm{~min}$. Incubate with the appropriate primary antibody diluted in blocking solution for $1 \mathrm{hr}$ at room temperature or overnight at $4{ }^{\circ} \mathrm{C}$. Wash fibers 3 times at 5 min per wash in PBS to remove any unbound antibody. Incubate with the appropriate secondary antibody for $45 \mathrm{~min}$ to $1 \mathrm{hr}$ at room temperature. Wash 3 times at 5 min per wash in PBS. Counterstain nuclei with $1 \mathrm{mg} / \mathrm{ml}$ DAPI. If staining floating fibers, transfer each fiber to a glass slide suitable for microscopy. Remove any excess of PBS or medium. Apply mounting medium and then add coverslip. Proceed to visualize myofibers under a fluorescence microscope. See Figure 4 for representative immunofluorescence on EDL myofibers. Alternative staining procedures using stronger fixatives are described in Verma, M. et al. ${ }^{11}$ and Wosniak, A.C. et al. ${ }^{12}$.

2. Oligonucleotide or plasmid transient transfection. Live myofibers can be transfected with plasmid or siRNA for specific gene/s of interest. When using siRNA, double transfection is recommended for efficient gene knockdown. We suggest performing the first transfection after 8 $\mathrm{hr}$ from the isolation. Six hr after the transfection, replace with fresh medium. For siRNA transfection, we suggest starting by using a final concentration of $50 \mathrm{nM}$. Gene expression knockdown can be analyzed by RNA extraction or preferably by immunostaining.

3. Viral infection. Infection of live myofibers is possible although the incidence of cell death is higher than with oligo transfection and the efficiency of infection may be variable depending on muscle conditions and age. For instance, intact adult muscle myofibers are particularly non responsive to viral infection due to the presence of the basal lamina which has been shown to provide a protective barrier against host infection ${ }^{13,14}$. Lentiviral vectors are preferred over retroviral vectors because they can infect quiescent (non mitotic) cells. For viral infection, it is advisable to culture fibers on a Matrigel coated dish or plate and let myofibers adjust to media conditions for the first $24 \mathrm{hr}$.

4. Live Imaging. Live imaging of myofibers is particularly time consuming and necessitates a microscope equipped with a $37{ }^{\circ} \mathrm{C}, 5 \% \mathrm{CO}_{2}$ chamber. It is useful when assessing the behavior of single satellite cells. Work done using this technique has been instrumental for the discovery of satellite cell heterogeneity and to study their behavior on fibers (15 and 16).

Representative Results

Here we described the isolation of single myofibers from the EDL muscle of adult mice. Successful myofibers yield depend on several factors such as collagenase activity or muscle conditions or age. Most importantly, the isolation of the muscle from tendon to tendon results in long, intact myofibers from EDL muscle. Figure 1 shows a step by step graphical representation of the "tendon to tendon" isolation. Once the muscle is fully digested, myofibers are released by applying gentle pressure to the muscle. Figure 2A shows a representative picture of a myofiber isolation experiment after the first wash. At this point the culture contains a mixture of bundles of single myofibers which appear as long and shining tubular structures, hyper contracted myofibers which are dark and short and debris from the digestion process. By the end of at least three consecutive washes, only single live myofibers should remain in the dish for culture or downstream analysis (Figure 2B). Figure 2C shows a long, live fiber as compared to a hyper contracted fiber $\left(C^{\prime}\right)$. At the time of the isolation, satellite cells appear as tiny protuberances on the myofiber surface (Figure 3A). If maintained in serum rich medium, satellite cells activate, proliferate, migrate and eventually fuse into myotubes (B). After 15 days in culture, all myotubes express the Myf5 reporter YFP (C) suggesting that muscle regeneration in the adult is performed by cells that at some point during their development had expressed the myogenic determinant factor Myf5. All satellite cells express the paired box transcription factor $\operatorname{Pax} 7^{17}$. The reporter mouse line Pax7Cre-TdTomato can be used to trace satellite cells via the expression of the TdTomato fluorescent signal (Figures 3D and E). Figure 4 shows a representative immunofluorescence of double Pax7+/Myod satellite cells. Classically double Pax $7+/$ Myod+ satellite cells are considered proliferative committed muscle progenitors which will either complete the differentiation program by down regulating Pax7 while maintaining Myod expression or return to quiescence by down regulating Myod and maintaining Pax7 expression $^{18}$ 

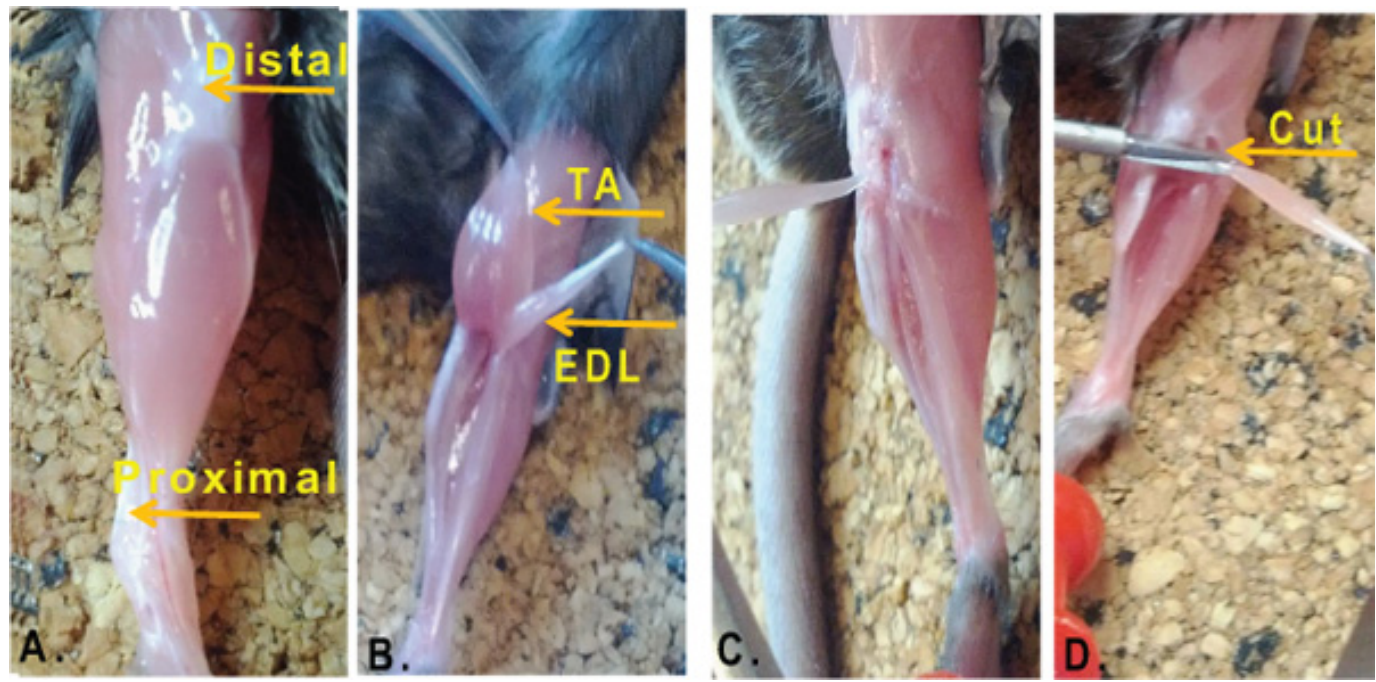

Figure 1. EDL muscle isolation from mouse hindlimb. A. Hindlimb of an 8 week old adult mouse. Arrows indicates the distal (knee) tendon and the proximal (foot) tendon. B. Anatomical position of the Tibialis Anterior (TA) muscle and the Extensor Digitorum Longus (EDL) muscle. C. By holding the EDL through the proximal tendon, the muscle is pulled toward the knee to expose the distal tendon. $\mathbf{D}$. The distal tendon is cut and the EDL is released.
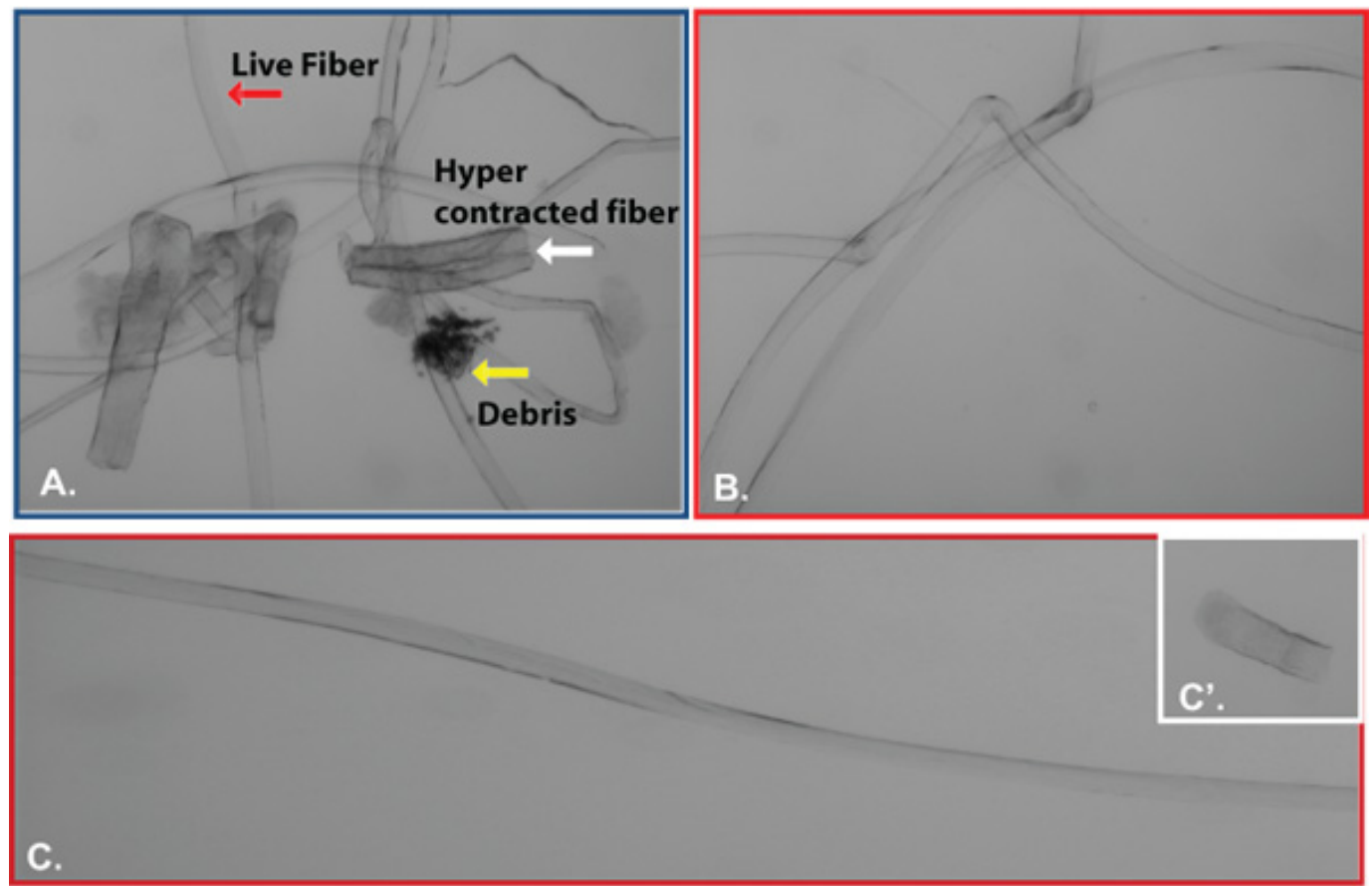

Figure 2. Representative results of a single myofiber isolation experiment. A . Bright field picture of a myofiber isolation experiment at the first wash step. Red arrow indicates live myofibers, white arrow indicates hyper contracted myofibers and yellow arrow indicates cells, myofiber or ECM debris. B. After consecutive washes in DMEM, only live myofibers remain. $\mathbf{C}$ and $\mathbf{C}^{\prime}$. Image represents a long intact live myofiber (C) and a short hyper contracted myofiber ('C'). Bar $10 \mu \mathrm{m}$. Pictures were taken with a Zeiss Axio Observer Z1 microscope equipped with AxioCam HR. 

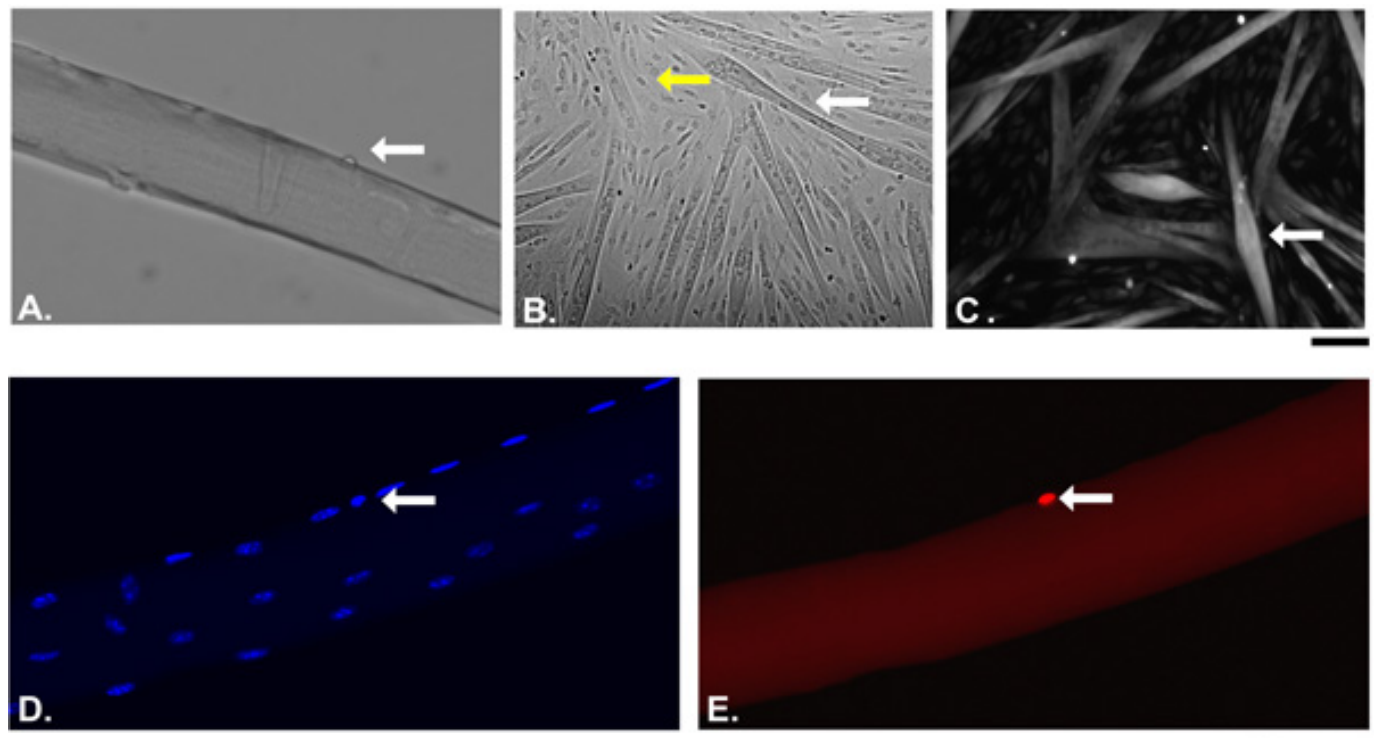

Figure 3. Representative results of a single myofiber culture experiment. A. Bright field picture of a single, live myofiber with its associated satellite cell (arrow) right after the isolation (Time 0). B. Single myofibers were plated on Matrigel and cultured in serum rich medium for 15 days. White arrow indicates differentiated myotubes as compared to single cells (yellow arrow) C. Single myofibers from a Myf5Cre;RosaYFP mouse were cultured as in B. Arrow indicates Myf5 derived myotubes. D and E. Single myofibers from Pax7Cre;TdTomato were isolated and fixed right after. Arrows indicate Pax7 positive quiescent satellite cell (E, red). Nuclei were counterstained with DAPI (D). Bar: $50 \mu \mathrm{m}$. Pictures were taken with a Zeiss Axio Observer Z1 microscope equipped with AxioCam HR.
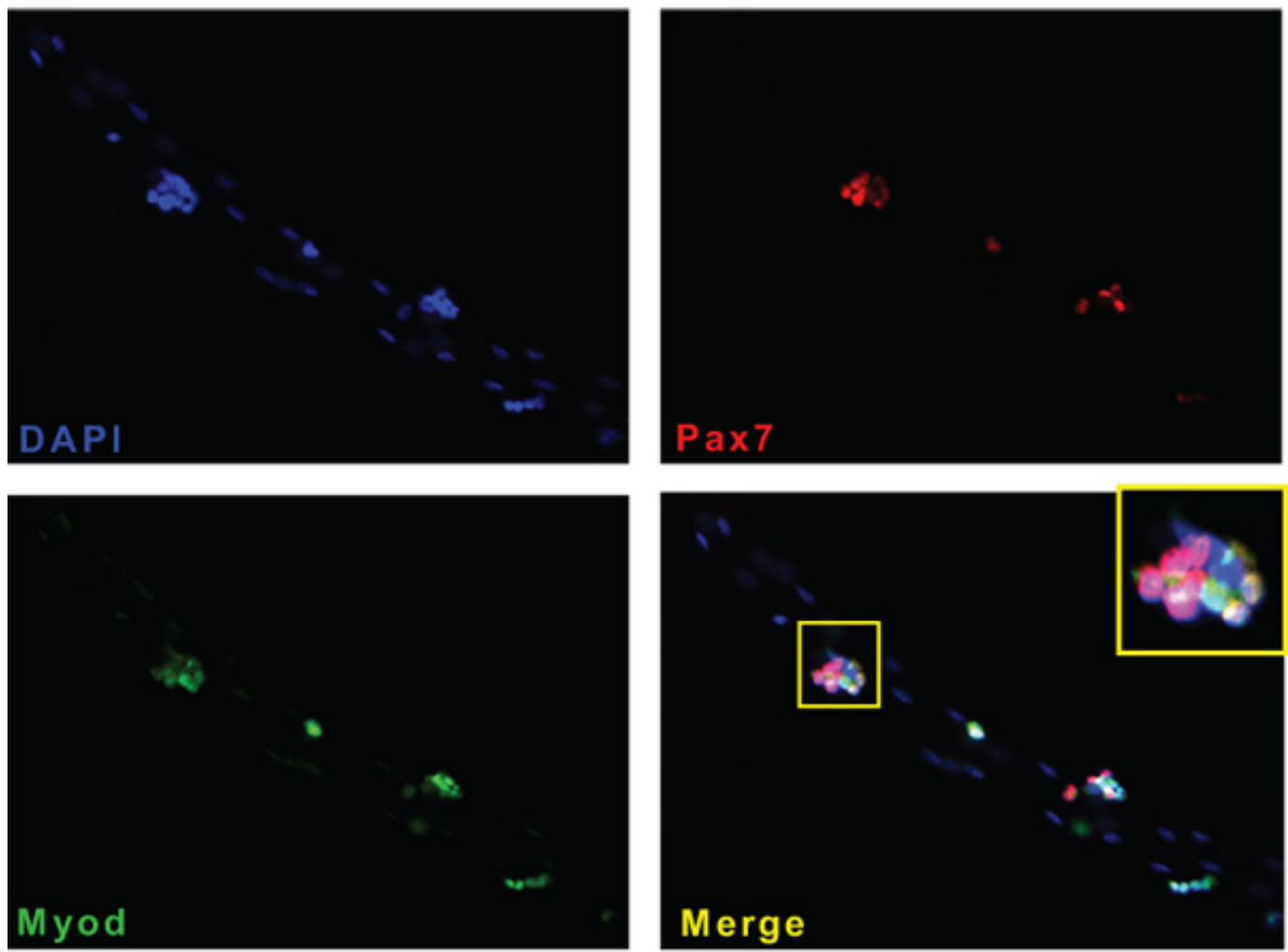

Figure 4. Example of immunofluorescence staining of satellite cells on myofibers. Single myofibers were isolated and cultured for $72 \mathrm{hr}$ in floating conditions. Satellite cells on myofibers were stained for the satellite cell specific marker Pax7 (B, red) and the myogenic regulatory factor Myod (C, green). Nuclei were counterstained with DAPI (A). Bars: $50 \mu \mathrm{m}$. Pictures were taken with a Zeiss Axio Observer Z1 microscope equipped with AxioCam HR. 


\begin{tabular}{|c|c|}
\hline Muscle Dissection (5 min each muscle) & $\begin{array}{ll}\text { - } & \text { Tendon to tendon isolation } \\
\text { - } & \text { Sharp tools } \\
\text { - } & \text { Minimal muscle damage }\end{array}$ \\
\hline Fiber isolation & $\begin{array}{l}\text { - Avoid over manipulation of fibers } \\
\text { - Eliminate debris with several washes }\end{array}$ \\
\hline Fiber Culture (Up to 3-4 weeks) & $\begin{array}{l}\text { - } \quad \text { Format: any } \\
\text { - } \quad \text { Coating: horse serum, Matrigel } \\
\text { - } \text { Medium: basal or high serum }\end{array}$ \\
\hline
\end{tabular}

Table 1. Overview of the myofiber isolation protocol. The myofiber isolation protocol consists of 4 major steps. For each step, the approximate time and major critical points are discussed. For detailed discussion of each step, refer to the protocol text.

\section{Discussion}

The isolation and culture of single myofibers from intact muscles provides an excellent in vitro model to study the process of muscle regeneration. A unique feature of this system is the preservation of satellite cells in their physiological environment beneath the basal lamina. Most importantly, the technique can be used to investigate muscle stem cell behavior in both quiescence and activated states. Over the past 20 years, the myofiber culture system has provided meaningful insights into the biology of the satellite cell population with respect to both intrinsic and extrinsic determinants. By culturing individual myofibers, satellite cell heterogeneity with respect to myofiber, muscle type or regenerative potential has been addressed ${ }^{15}$. Elaborate studies using live imaging of single cultured fibers allowed for the gaining information on satellite cell migration pattern ${ }^{16}$. The acquisition of quantitative data is also possible. Although time consuming, it provides significant information on the distribution and occurrence of specific events (stem cells asymmetric division, proliferation and differentiation ratio, etc.). Together with previously described applications, protein or RNA extraction from single fibers is also possible, although isolation of pure population of satellite cells by FACS or other means may provide a better platform to investigate protein or gene expression changes at a molecular level. In our experience, the most critical step for successful fiber isolation is the "tendon to tendon" isolation (steps 2.5 to 2.9 in the protocol text). This guarantees that after muscle digestion, fibers are released from the EDL with minimal or no damage thus increasing their performance in the following steps.

\section{Disclosures}

No competing interests.

\section{Acknowledgements}

We would like to thank Sarah Dick for providing critical reading and comments. M.A.R. holds the Canada Research Chair in Molecular Genetics and is an International Research Scholar of the Howard Hughes Medical Institute. This work was supported by grants to MAR from the National Institutes of Health, the Howard Hughes Medical Institute, the Canadian Institutes of Health Research, the Muscular Dystrophy Association and the Canada Research Chair Program.

\section{References}

1. Bischoff, R. Proliferation of muscle satellite cells on intact myofibers in culture. Dev. Biol. 115 (1), 129-139 (1986).

2. Rosenblatt, J.D., Lunt, A.I., Parry, D.J., \& Partridge, T.A. Culturing satellite cells from living single muscle fiber explants. In Vitro Cell Dev. Biol. Anim. 31 (10), 773-9 (1995).

3. Anderson, J.E., Wozniak, A.C., \& Misunoya W. Single muscle fiber isolation and culture for cellular molecular, pharmacological, and evolutionary studies. Methods Mol. Biol. 798, 85-102, Humana Press, New York, NY, 10013, USA, (2012).

4. Shefer, G. \& Yablonka-Reuveni, Z. Isolation and culture of skeletal muscle myofibers as a means to analyze satellite cells. Methods Mol. Biol. 290, 281-304, Human Press Inc, Totowa, NJ, (2005).

5. White, R.B., Biérinx, A.S., Gnocchi,V.F., \& Zammit, P.S. Dynamics of muscle fibre growth during postnatal mouse development. BMC Developmental Biology. 10 (21), 1-11 (2010).

6. Siegel, A.L., Kuhlmann, P.K., \& Cornelison, D.D. Muscle satellite cell proliferation and association: new insights from myofiber time-lapse imaging. Skeletal Muscle. 2 (1), 1-7 (2011).

7. Pasut, A., Oleynik, P., \& Rudnicki, M.A. Isolation of muscle stem cells by fluorescence activated cell sorting cytometry. Methods Mol. Biol. 798, 53-64, Humana Press, New York, NY, 10013, USA, (2012).

8. Engler, A.D., Griffin, M.A., Sen, S., Bonnemann, C.G., Sweeney, H.L., \& Discher, D.E. Myotubes differentiate optimally on substrates with tissue-like stiffness: pathological implications for soft or stiff microenvironments. J. Cell. Biol. 166 (4), 877-87 (2004).

9. Boonen, K.J. \& Post, M.J. The muscle stem cell niche: regulation of satellite cells during regeneration. Tissue Eng. Part B Rev. 14 (4), $419-31$ (2008).

10. Wozniak, A.C. \& Anderson, J.E. Single-fiber isolation and maintenance of satellite cell quiescence. Biochem. Cell Biol. 83 (5), 674-6 (2005). 
11. Verma, M. \& Asukura, A. Efficient Single Muscle Fiber Isolation from Alcohol-Fixed Adult Muscle following $\beta$-Galactosidase Staining for Satellite Cell Detection. J. Histochem. Cytochem. 59 (1), 60-7 (2001).

12. Wosniak, A.C., Pilipowics, O., et al. C-Met expression and mechanical activation of satellite cells on cultured muscle fibers. J. Histochem. Cytochem. 51 (11), 1437-45 (2003).

13. Huard, J., Feero, W.G., Watkin, S.C., Hoffman, E.P., Rosenblatt, J.D., \& Glorioso, J.C. The basal lamina is a physical barrier to herpes simplex virus-mediated gene delivery to mature muscle fibers. J. Virol. 70 (11), 8117-23 (1996).

14. Feero, W.G., Rosenblatt, J.D., et al. Viral gene delivery to skeletal muscle: insights on maturation-dependent loss of fiber infectivity for adenovirus and herpes simplex type 1 viral vectors. Hum. Gene Ther. 8 (4), 371-80 (1997).

15. Kuang, S., Kuroda, K., Le grand, F., \& Rudnicki, M.A. Asymmetric self-renewal and commitment of satellite stem cells in muscle. Cell. 129 (5), 999-1010 (2007).

16. Siegel, A.L, Atchison, K., Fisher, K.E., Davis, G.E., \& Cornelison, DD. 3D timelapse analysis of muscle satellite cell motility. Stem Cells. 27 (10), 2527-38 (2009).

17. Seale, P., Sabourin, L.A., Girgis-Gabardo, A., Mansouri, A., Gruss, P., \& Rudnicki, M.A. Pax7 is required for the specification of myogenic satellite cells. Cell. 102 (6), 777-86 (2000).

18. Zammit, P., Golding, J.P., Nagata, Y., Hudon, V., Partridge, T.A., \& Beauchamp J.R. Muscle satellite cells adopt divergent fates: a mechanism for self-renewal? J. Cell Biol. 166 (3), 347-57 (2004). 